\title{
FLOW STRUCTURE AND TURBULENCE IN NEAR FIELDS OF CIRCULAR AND NONCIRCULAR JETS
}

\section{N. E. Öçer, G. Taşar, O. Uzol, and S. Özgen}

Aerospace Engineering Department

Middle East Technical University

Ankara 06531, Turkey

\begin{abstract}
Experimental investigation of the turbulent flow field of jets emanating from circular and curved-edged noncircular nozzles is conducted using hotwire anemometry. Distributions of the mean velocity vector are obtained by traversing a triple-sensor hot-wire probe at varying streamwise locations downstream of the jet exit plane in a low-speed free-jet facility. Measurements are obtained for a baseline circular nozzle (round jet) and for equilateral triangular and square nozzles. The data are used to compare the structure of the flow field within these jets such as their mean velocity, turbulent kinetic energy, and entrainment levels. Axis rotation phenomenon is also investigated for noncircular nozzles, and it was not observed up to a streamwise location of $10 d$ ( $d$ is the nozzle equivalent diameter, equals to $40 \mathrm{~mm}$ ) for noncircular nozzles.
\end{abstract}

\section{INTRODUCTION}

Mixing helps to convert heterogeneous physical systems into more homogeneous ones. For example, in processes such as combustion, in which chemical reactions between the fuel and the oxidizer are accompanied by the production of heat, mixing becomes a complex problem that is usually difficult to define and analyze. The mixing of air and fuel ejecting from nozzles needs special attention. Improved combustion performance may be obtained by using noncircular nozzle geometry as a passive mixing control technique. Ejection of a jet through a nozzle into stagnant ambient air constitutes a general configuration for numerical and experimental mixing studies. Vorticity distribution, which influences the evolution of turbulent structures in the flow, is an important parameter that can be used to understand the mixing levels. Entrainment of the ambient fluid is generally driven by large and small scale turbulent structures within the jet flow field [1].

This is an Open Access article distributed under the terms of the Creative Commons Attribution-Noncommercial License 3.0, which permits unrestricted use, distribution, and reproduction in any noncommercial medium, provided the original work is properly cited. 
Various researchers have investigated circular and noncircular sharp edged orifices and jets. In the numerical study of Miller et al. [2], three-dimensional jets emanating from noncircular nozzles were visualized and compared with circular ones at low Reynolds numbers using Direct Numerical Simulation (DNS) method. The axis rotation phenomenon, an indicator of better mixing, was observed for all noncircular jets. The work by Gutmark and Grinstein [3] has also indicated the significance of axis rotation of noncircular jets, which make them more efficient mixers than the circular jets. The jet core length was also stressed as a measure of mixing, and the results showed that the isosceles triangular jet is the most efficient mixer over other jets investigated. On the experimental side, Quinn [4] has investigated the near-field of a flow emanating from a sharp-edged equilateral triangular orifice by taking velocity measurements with a hot-wire anemometer. As a result, some quantitative results on the mixing rate were obtained and the equilateral triangular jet was found to be a better mixer compared to the circular one. One point that is not fully investigated in previous studies is the effect of corner radii in noncircular jets and this point is indicated to be a further research topic by Quinn [4]. The existence of sharp corners in the nozzle can increase the fine-scale turbulence at the corners relative to the flat segments of the nozzle as indicated in [5, 6], and enhance mass entrainment significantly in [7].

In this study, mean velocity and turbulence fields of jets emanating from circular and noncircular nozzles with corner radii are investigated experimentally using hotwire anemometry. Experiments were performed in a low-speed free-jet facility with a maximum mean streamwise velocity of $22 \mathrm{~m} / \mathrm{s}$. The corresponding Reynolds number is $6 \cdot 10^{4}$ based on the mean streamwise velocity and the nozzle equivalent diameter $(d=40 \mathrm{~mm})$. Distributions of all three components of the mean velocity vector as well as all six components of the Reynolds stress tensor are obtained on vertical measurement planes that are parallel to the nozzle exit plane by traversing a triple-sensor hot-wire probe at various streamwise locations downstream of the jet exit plane. A series of longitudinal measurements were also taken along the centerlines of the jets covering a region from $x / d=0$ to 10 to get a better understanding of potential core lengths of the jets and variations in turbulence levels. Measurements are obtained for a baseline circular nozzle (round jet) and for equilateral triangular and square nozzles. The data are used to compare the structure of the flow field within these jets such as their decay, entrainment rate, and turbulent kinetic energy levels.

\section{EXPERIMENTAL SETUP AND METHODOLOGY}

The measurements are conducted in a free-jet facility shown in Fig. 1. It consists of an axial fan, a diffuser, a settling chamber, a contraction, and a transition duct on which pipe nozzles with different cross-sectional shapes can be attached. 
The diffuser, settling chamber, and contraction are constructed from Plexiglas, whereas the fan transition duct and nozzles are made of metal. The diffuser has an inlet area of $0.5 \times 0.66 \mathrm{~m}$ and an exit area of $0.66 \times 0.66 \mathrm{~m}$ and is $0.34 \mathrm{~m}$ in length. The settling chamber is a square cube having a length of $0.565 \mathrm{~m}$. The contraction has a square cross-sectional area with a length of $0.29 \mathrm{~m}$ and has inlet and exit areas of $0.66 \times 0.66 \mathrm{~m}$ and $0.237 \times 0.237 \mathrm{~m}$, respectively, resulting in a contraction ratio of 7.75 . After the contraction, different transition ducts are used to convert the cross-sectional geometry into the required shape within a distance of $0.5 \mathrm{~m}$. Three different pipe nozzles are used that have the cross-sectional shapes of circular, square, and equilateral triangle with a length of $0.5 \mathrm{~m}$ and a jet exit equivalent diameter of $d=40 \mathrm{~mm}$. By using an automated traversing mechanism, which has a maximum resolution of $25.4 \mu \mathrm{m}$ in each direction, the three components of the velocity vector are acquired at five different streamwise stations (i. e., $x / d=1,2,4,6$, and 10). The probe was traversed through a total length of $152.4 \mathrm{~mm}$ on vertical planes with a grid spacing of $2.54 \mathrm{~mm}$ in $y$ and $z$ directions. In the case of longitudinal measurements, data are collected along the centerline $(y=0)$ of the three jets from $x / d=0$ to 10 , through a total length of $394.34 \mathrm{~mm}$ in $x$ direction and $148.59 \mathrm{~mm}$ in $z$ direction with a grid spacing of $5.715 \mathrm{~mm}$ in both directions.

Figure 1 shows the coordinate system and the flow facility that is used in the study. Honeycombs and screens were used at the settling chamber to obtain a homogeneous flow across the facility and pads were used at necessary points to prevent the facility to vibrate. The measurement unit in the experiments is a DANTEC 55P91 tri-axial probe which has three mutually perpendicular gold-plated sensors providing the necessary information for calculation of the

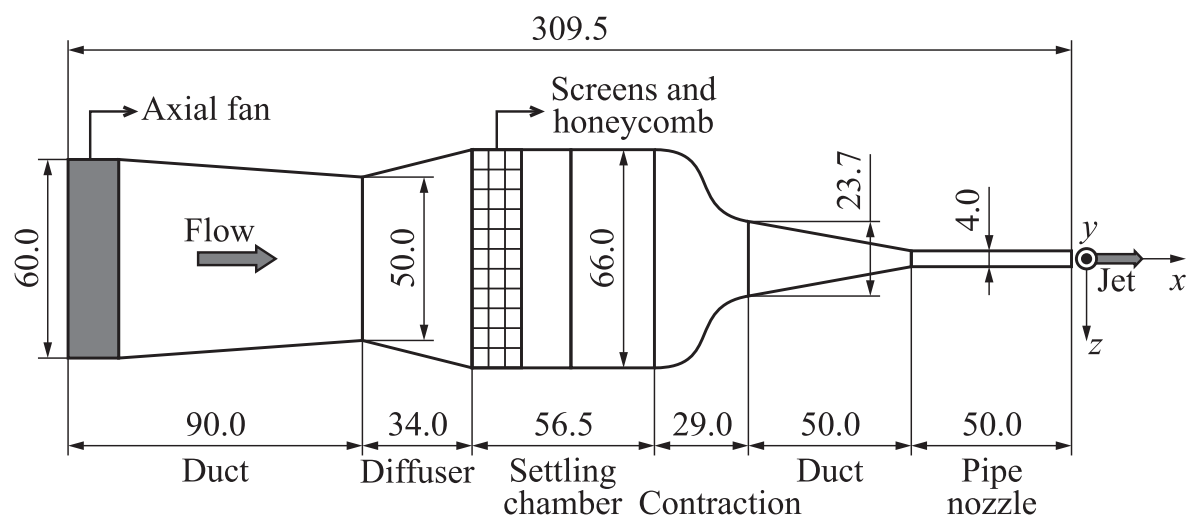

Figure 1 Free jet flow facility and the jet coordinate system. Dimensions are in centimeters 


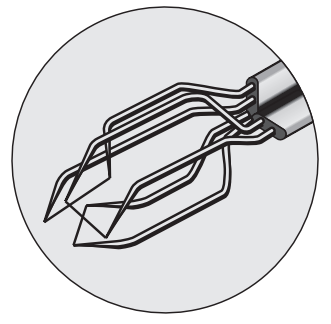

(a)

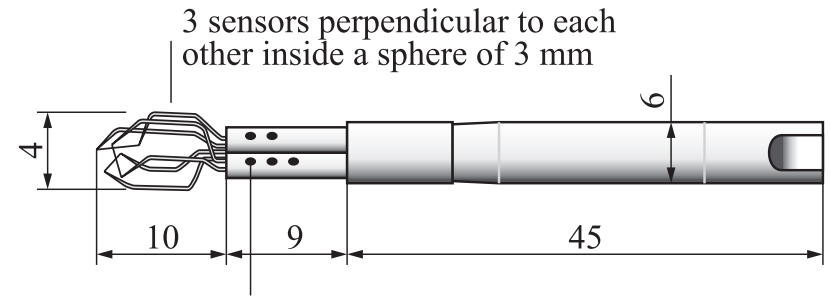

(b)

Figure 2 DANTEC 55P91 tri-axial hotwire anemometer (image is taken from [8]. Dimensions are in millimeters

full Reynolds stress tensor. As shown in Fig. 2, three 5-micrometer diameter sensors are perpendicular to each other inside a sphere of 3-millimeter diameter which corresponds to $7.5 \%$ of equivalent jet diameter.

The probe is driven by a DANTEC 54 N81 multichannel Constant Temperature Anemometry (CTA) module. The data acquisition from the hot-wire module is performed using a NI 9205 analog input module that has a $250 \mathrm{kS} / \mathrm{s}$ aggregate sampling rate. Data are sampled at $5 \mathrm{kHz}$ for $2 \mathrm{~s}$ resulting in 10,000 samples at each grid point. LabView is used for the coordination of the traverse mechanism and the CTA module. Sampling rate, number of samples, sampling duration, and grid resolution are all set by the LabView code. The manufacturer's given constants are used for the directional calibration, and the velocity calibration is conducted at the center point of the exit of the circular nozzle. An MMF (Morgan-Mercer-Flodin) model is used to convert the CTA output voltage into velocity values. This model is the nonlinear regression model producing sigmoidal or "S-shaped" growth curves. These curves start at a fixed point and increase monotonically to reach an inflection point. After this, the growth approaches a final value asymptotically. In this model, the output, $y$, is expressed in terms of input, $x$, by the following relation:

$$
y=\frac{a b+c x^{d}}{b+x^{d}}
$$

where $a, b, c$, and $d$ are the fitting constants.

The following are the equations which convert the CTA output voltages $e_{1}$, $e_{2}$, and $e_{3}$ into the calibration velocities, namely, $U_{\text {cal1 }}, U_{\text {cal2 }}, U_{\text {cal3 }}$ for $20^{\circ} \mathrm{C}$ :

$$
U_{\text {cal1 }}=\frac{-0.4439668 \cdot 400.47362+107.14277 e_{1}^{7.9047463}}{400.47362+e_{1}^{7.9047463}}
$$




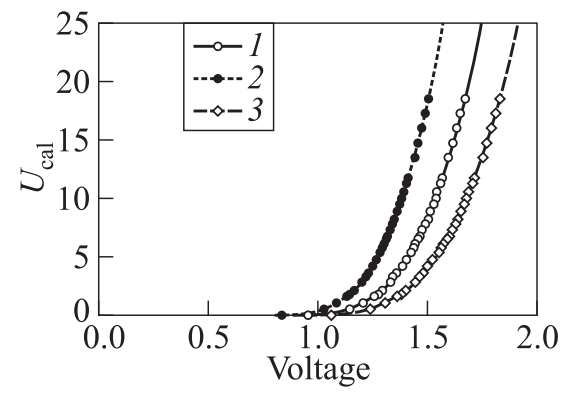

Figure 3 Calibration curves for $20{ }^{\circ} \mathrm{C}: 1-e_{1}$ vs. $U_{\text {cal1 }} ; 2-E_{2}$ vs. $U_{\text {cal } 2}$; and $3-$ $E_{3}$ vs. $U_{\text {cal } 3}$

$$
\begin{aligned}
& U_{\text {cal2 }}=\frac{-0.53443656 \cdot 232.62451+155.85194 e_{2}^{7.2805617}}{232.62451+e_{2}^{7.2805617}} ; \\
& U_{\text {cal3 }}=\frac{-0.49409868 \cdot 903.65461+164.77246 e_{3}^{7.4223736}}{903.65463+e_{3}^{7.4223736}}
\end{aligned}
$$

The calibration curves are shown in Fig. 3.

\section{RESULTS}

Before presenting the near field characteristics, Fig. 4 shows the jet exit conditions normalized by local maximum velocity of the corresponding jet for the three different nozzle geometries just downstream of the nozzle exit plane at about $x / d=0.025$. The mean axial velocity and the turbulent kinetic energy distributions, which are nondimensionalized by $U_{j}$ and $U_{j}^{2}$, respectively, are presented in the figure and the main flow direction is into the page. Here, $U_{j}$ is taken as the average mean axial velocity for each profile. As is evident, all three geometries have very similar exit conditions both in terms of mean velocity as well as the turbulent kinetic energy. The highest turbulence levels coincide with the highest shear regions at the edges of the jets as expected, which also correspond to the wall boundary layer regions inside the nozzle pipes. Since the pipe nozzles are not long enough to establish a fully developed flow within the pipes, there still exists a low turbulence potential core region in all jets, with the triangular having the smallest among the three.

Figure 5 shows the distributions of mean streamwise (i.e., $x$-component) velocity for circular, square, and triangular nozzles at five different streamwise locations. These distributions are nondimensionalized using the jet exit velocity, $U_{j}$, in order to highlight the differences in the decay of the potential core region. 


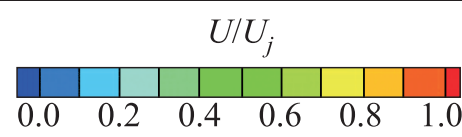

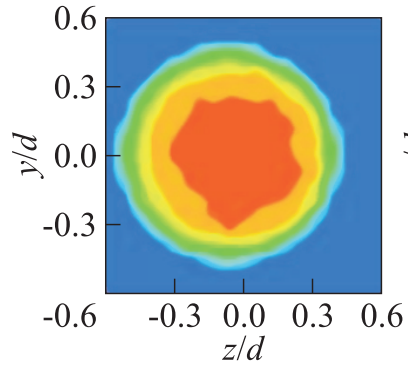

$z / d$

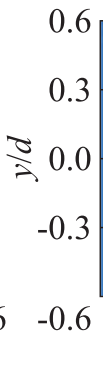

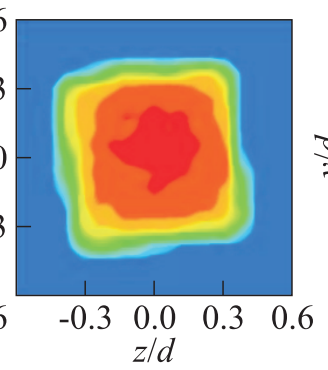

(a)

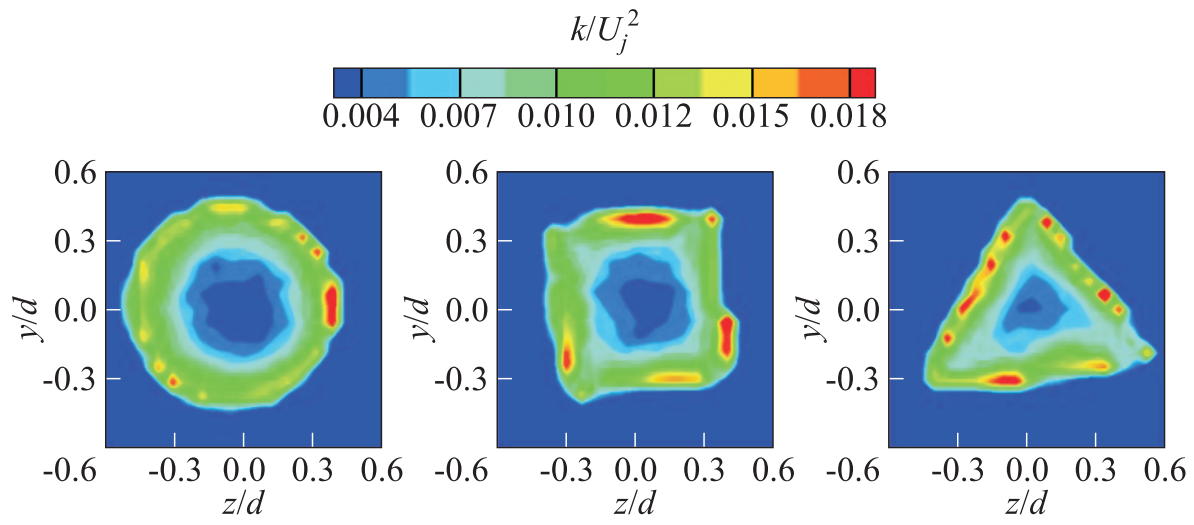

(b)

Figure 4 Mean axial velocity, $U(a)$, and turbulent kinetic energy, $k(b)$, distributions just downstream of the jet exit plane $(x / d=0.025)$ for the circular (left), square (middle), and triangular (right) jets. (Refer Öçer et al., p. 46.)

It can be observed that initial axes orientation of triangular and square jets are conserved along the streamwise direction, that is, contrary to the previous investigations on sharp-edged noncircular jets, no axis-switching phenomenon is observed up to $10 \mathrm{~d}$ for these curved-edge nozzles. The diffusion of all three types of jets is clearly evident as the jet moves downstream. However, the triangular jet seems to be diffused more when one compares the relative sizes of velocity fields for all three types of jets, for example, at $10 d$, and this, surely, is an indicator of enhanced mixing at this location.

However, a more quantitative analysis on mixing can be achieved by calculating entrainment rates using these velocity profiles. The entrainment rates for the nozzles are shown in Fig. 6. These are calculated using the following expression: 

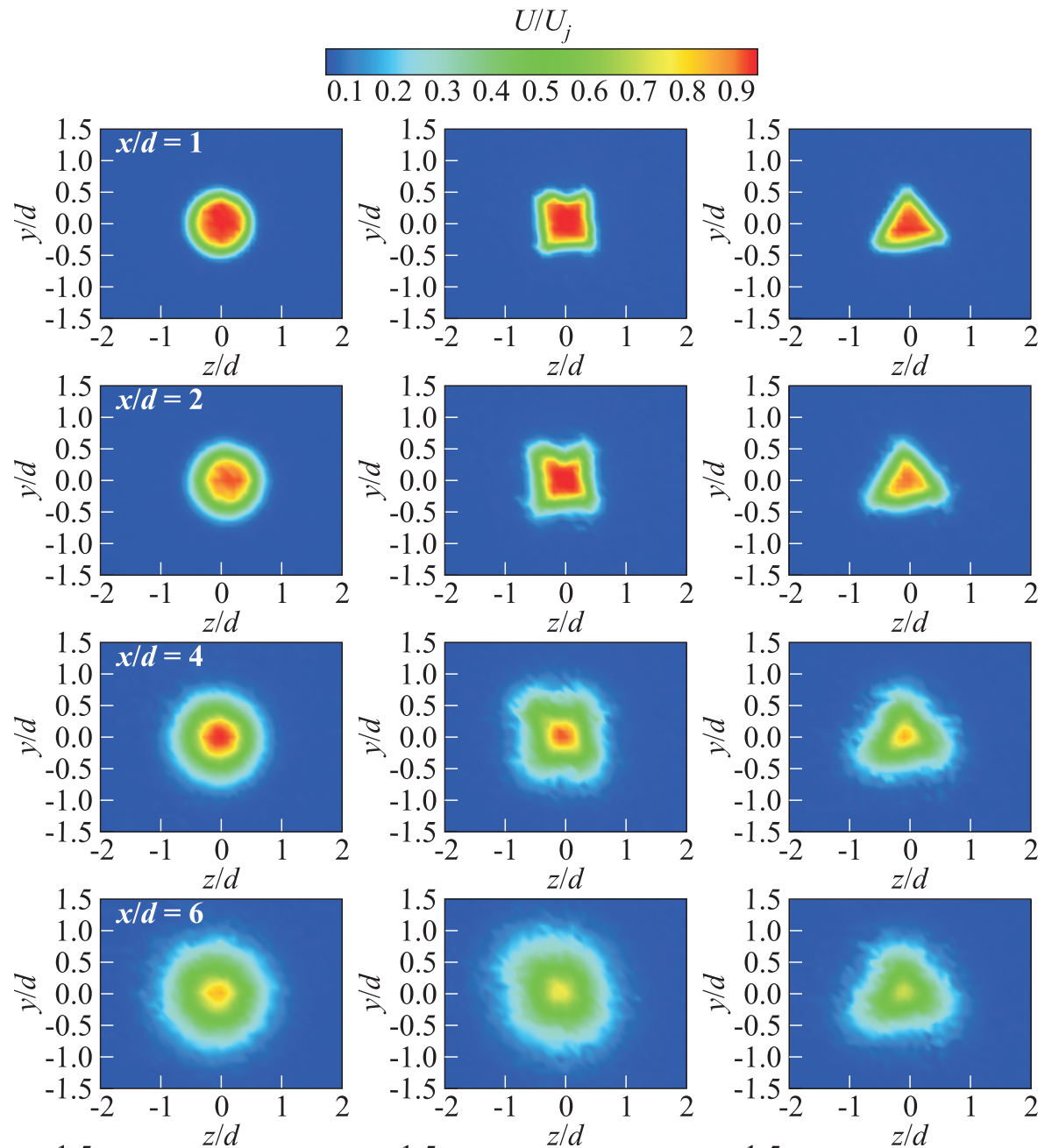

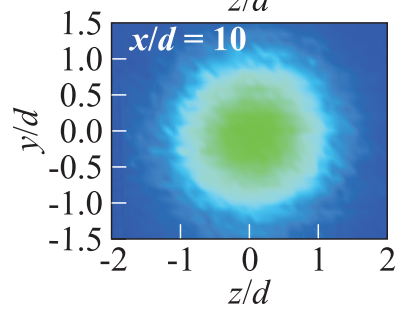

(a)

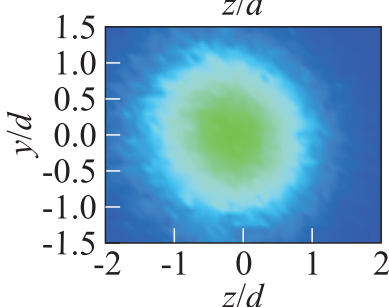

(b)

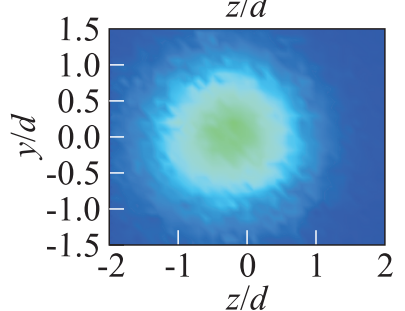

(c)

Figure 5 Mean streamwise velocities nondimensionalized by jet exit velocities at various streamwise locations $(x / d=1,2,4,6$, and 10) for circular $(a)$, square $(b)$, and triangular (c) jets. (Refer Öçer et al., p. 47.) 


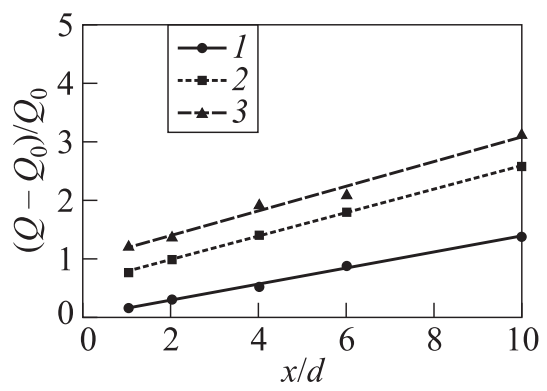

Figure 6 Entrainment rates for circular (1), square (2), and triangular (3) jets

$$
\text { Mass entrainment rate }=\frac{Q-Q_{0}}{Q_{0}}
$$

where $Q_{0}$ is the mass flux at jet exit and $Q=\sum_{j} \rho V_{j} \Delta A_{j}$.

Entrainment rate values never take negative values in the region of investigation, as opposed to the results presented by Quinn [4] at an orifice exit. However, regardless of the nonexistence of axis-switching, the results shown in Fig. 6 are consistent with those of Quinn as the triangular jet introduces the highest mass entrainment rate when compared with round and square jets.

Another quantity that is an effective factor in mixing is the turbulent kinetic energy, $k$. Turbulent kinetic energy is the mean kinetic energy per unit mass in the fluctuating velocity field and it is a measure of interaction between the flow and the ambient air, such that higher $k$ values mean higher interaction or mixing. The turbulent kinetic energy distributions as normalized by the nozzle exit velocities, shown in Fig. 7, indicate that the turbulent kinetic energy levels within the jet flow structure rise much quicker for triangular and square jets compared to the round jet. On the other hand, the decay of $k$ has the highest value for triangular jet and the lowest value for the round jet. The fact that the turbulent kinetic energy levels are increasing with downstream distance is consistent with the results provided by Quinn [9].

In order to better comprehend and visualize the velocity decay and contraction of the potential core, longitudinal measurements are conducted at the center plane of each type of jet flow. Gutmark and Grinstein [3] stated that the higher mixing rate results in a smaller potential core. Together with this information and referring to Fig. 8 in which the triangular jet has the smallest potential core, it can be said that triangular jet has the higher mixing rate.

The turbulent kinetic energy distributions on longitudinal planes are presented in Fig. 9. It can be observed that turbulent kinetic energy is generated on high shear regions on either side of the jet for all geometries. The levels keep increasing in the streamwise direction within the shear layers and then the two 

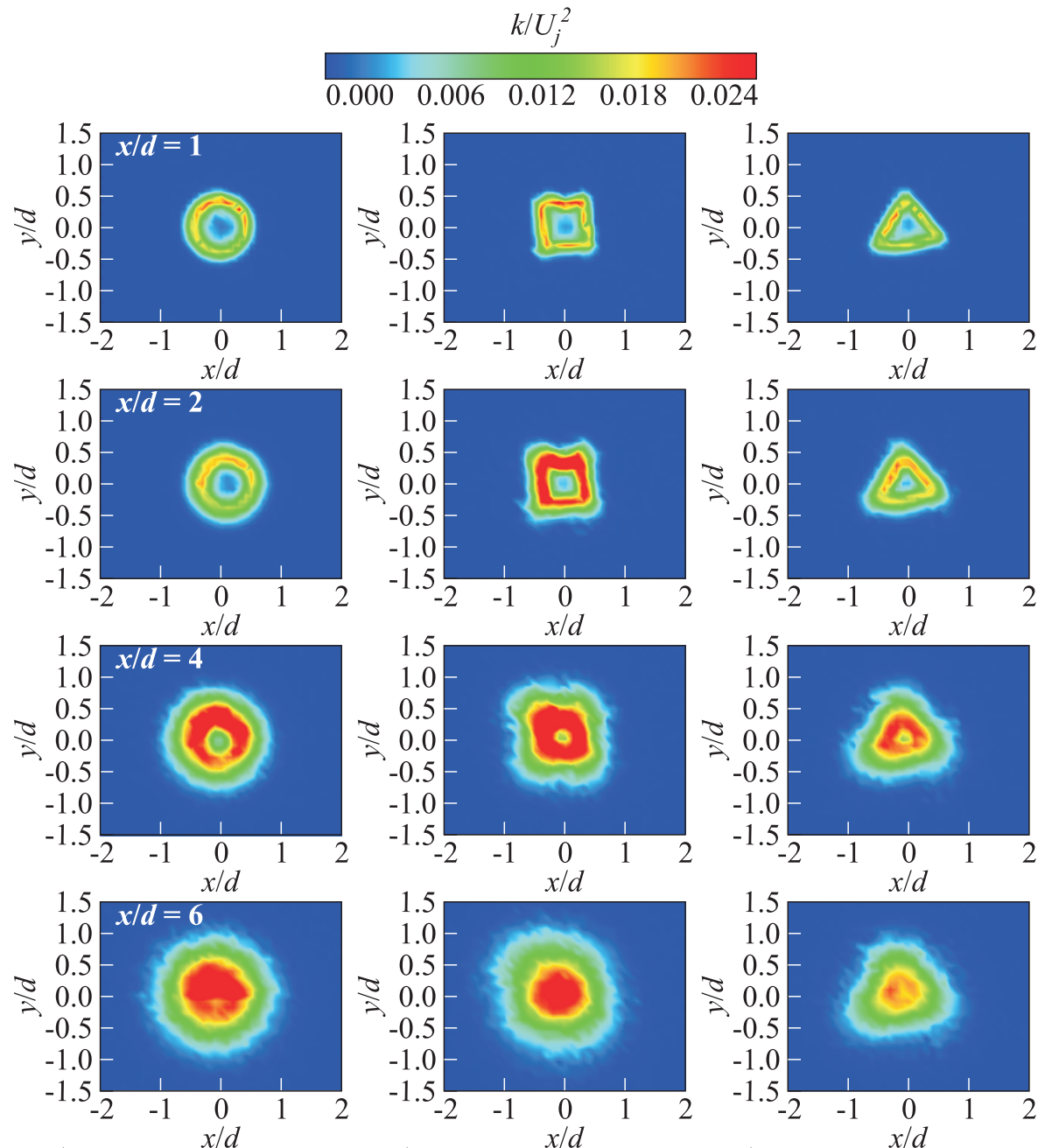

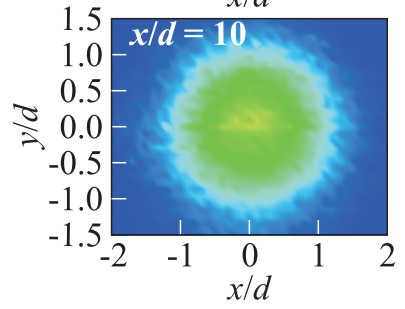

(a)

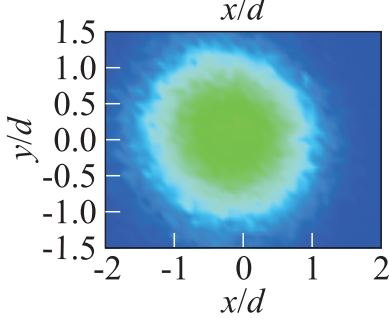

(b)

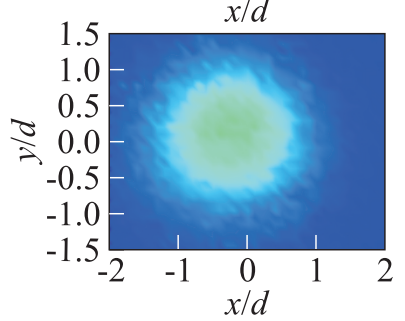

(c)

Figure 7 Normalized turbulent kinetic energy field $\left(k / U_{j}^{2}\right)$ at various data acquisition stations for different nozzle geometries: (a) circular; (b) square; and (c) triangular. (Refer Öçer et al., p. 49.) 


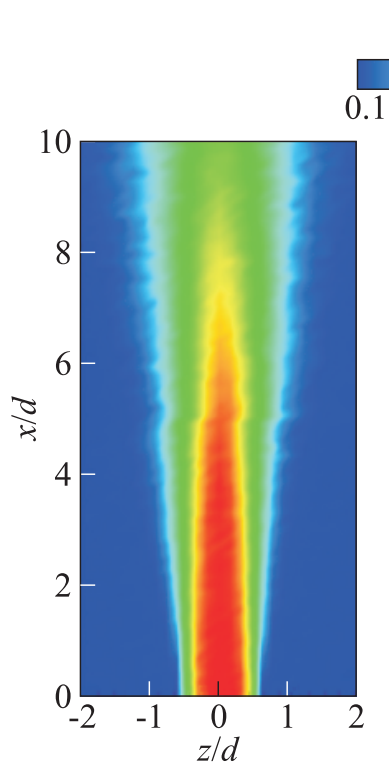

(a)

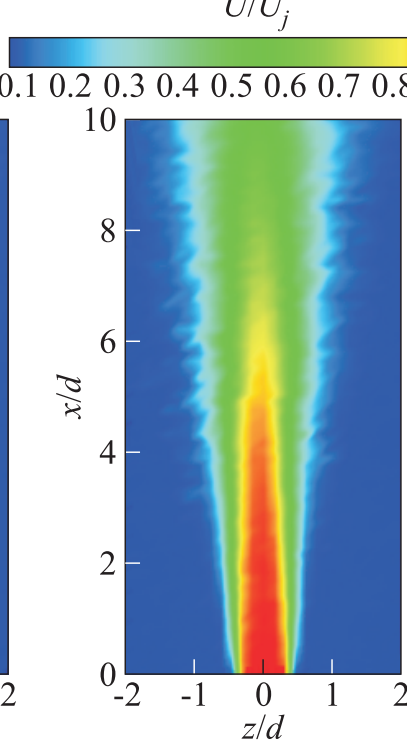

(b)

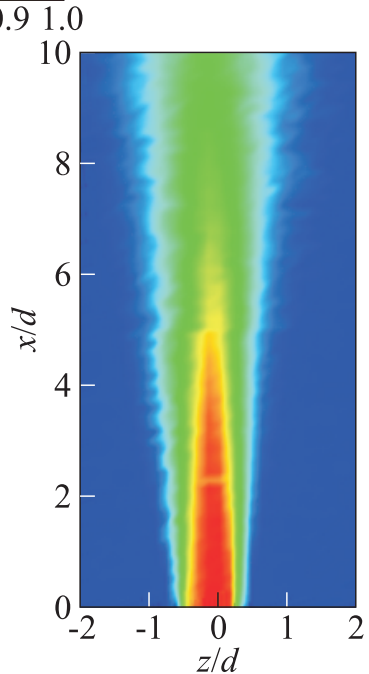

(c)

Figure 8 Normalized streamwise mean velocity fields, $\bar{U} / U_{j}$, on longitudinal planes: (a) round; (b) square; and (c) triangular jets. (Refer Öçer et al., p. 50.)

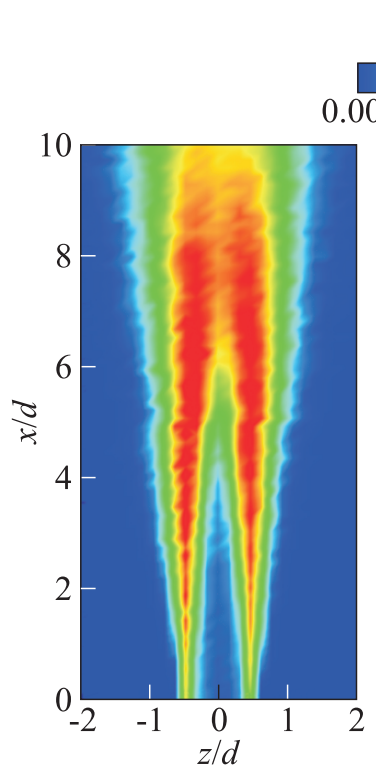

(a)

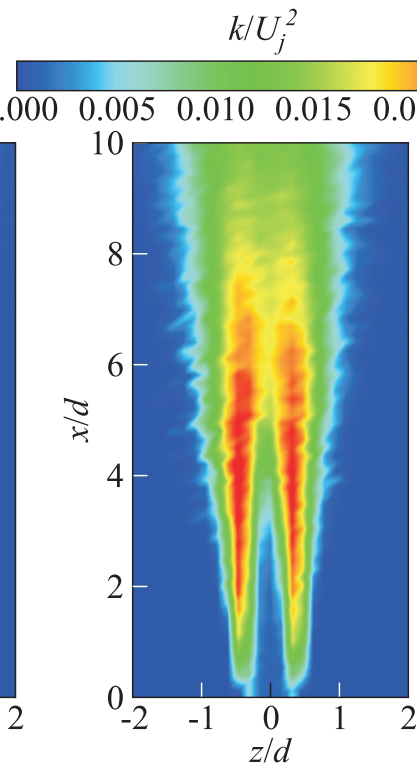

(b)

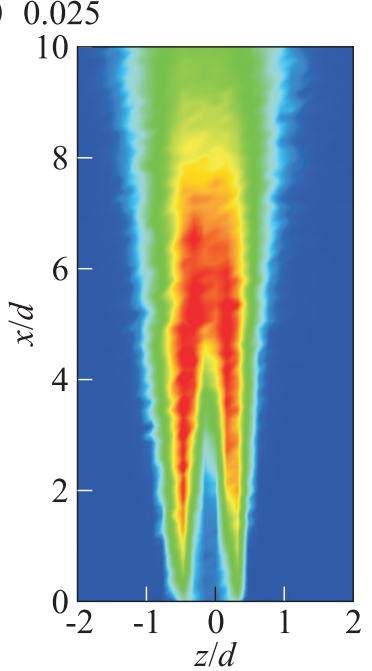

(c)

Figure 9 Normalized longitudinal turbulent kinetic energy field, $k / U_{j}^{2}$, on longitudinal planes: ( $a$ ) round; (b) square; and $(c)$ triangular jets. (Refer Öçer et al., p. 50.) 
high turbulent kinetic energy regions on either side of the jet centerline merge further downstream. The merging occurs earliest in the case of the triangular jet at about $x / d=4.5$. The circular jet has the latest merging of the two regions at about $x / d=6$. The square jet layers merge a little earlier than the circular jet. Relatively high turbulent kinetic energy levels around the centerline in the near field of the triangular jet, probably, are the main reasons behind the quick disappearance of the potential core region due to enhanced mixing.

\section{CONCLUDING REMARKS}

An experimental study was conducted to investigate the turbulence structures as well as the mixing levels in the near fields of jets emanating from circular, triangular, and square nozzles with corner radii using constant temperature hotwire anemometry. The results are compared with those obtained from a baseline round jet. The axis switching phenomenon was not observed within the investigated region for all the jets. The entrainment rate value obtained from mean velocity profile is found to be the highest for the triangular and the lowest for the round jet in the investigation region. The turbulent kinetic energy distributions of all three types of jets indicate that the turbulent kinetic energy levels within the jet flow structure rise and decay much quicker for triangular jet, comparing with round and square ones. Moreover, the results from measurements in the longitudinal direction made it clear that triangular jet flow has the shortest core length and higher turbulence level compared to other two jet flows. In the light of these results, it is evident that a triangular jet flow possesses better mixing characteristics such as the highest mixing rate, mass entrainment rate, and turbulence level over round or square ones.

\section{ACKNOWLEDGMENTS}

The support of Scientific and Technical Research Council of Turkey (TÜBİTAK) for this study is greatly acknowledged (project No.106M067).

\section{REFERENCES}

1. Gutmark, E. J., K. C. Schadow, T. P. Parr, D. M. Hanson-Parr, and K. J. Wilson. 1989. Noncircular jets in combustion systems. Exper. Fluids 7:248-58.

2. Miller, R. S., C. K. Madnia, and P. Givi. 1995. Numerical simulation of non-circular jets, Computers Fluids 24(1):1-25. 
3. Gutmark, E. J., and F. F. Grinstein. 1999. Flow control with non-circular jets. Ann. Rev. Fluid Mech. 31:239-72.

4. Quinn, W.R. 2005. Near-field measurements in an equilateral triangular turbulent free jet. AIAA J. 43(12):2574-85.

5. Schadow, K. C., E. J. Gutmark, D. M. Parr, and K. J. Wilson. 1988. Selective control of flow coherence in triangular jets. Exper. Fluids 6:129-35.

6. Toyoda, K., and A. K. M. F. Hussain. 1989. Vortical structures of noncircular jets. 4th Asian Congress Fluid Mech.. Hong Kong. A117-127.

7. Vandsburger, U., and C. Ding. 1995. The spatial modulation of a forced triangular jet. Exper. Fluids 18:239-48.

8. http://www.dantecdynamics.com/Default.aspx?ID=767\#1788.

9. Quinn, W. R. 2005. Measurements in the near flow field of an isosceles triangular turbulent free jet. Exper.s Fluids 39:111-26. 